Check for updates

Cite this: RSC Adv., 2019, 9, 10282

\title{
Ethylene glycol-based solar-thermal fluids dispersed with reduced graphene oxide $\uparrow$
}

\author{
Lei Shu, Jingyi Zhang, Benwei Fu, Jiale Xu, Peng Tao, D * Chengyi Song, \\ Wen Shang, Jianbo Wu (DD and Tao Deng (D)*
}

Direct absorption solar collectors, which use optical nanofluids to volumetrically absorb and convert sunlight into heat, have emerged as promising devices to harvest solar-thermal energy for many heat-related applications. Nanofluids, however, generally suffer from aggregation issues and the widely investigated water-based fluids only enable solar-thermal harvesting at relatively low temperatures. Herein, we report a facile way to prepare stably dispersed reduced graphene oxide-ethylene glycol ( $\mathrm{rGO}-\mathrm{EG}$ ) fluids for solar-thermal energy harvesting at medium temperatures. Without the use of complex surface modification process, the homogeneous dispersion of rGO-EG fluids was achieved by utilizing the favorable interaction between the oxygencontaining groups on the rGO surfaces and EG molecules. The rGO-EG fluids were prepared by reducing the GO-EG fluids that are uniformly dispersed with ethanol-wetted GO through a single step of heating. The prepared rGO-EG fluids have suitable thermophysical properties for direct solar-thermal energy harvesting, such as broadband absorption of sunlight, high specific heat capacity and low viscosity. The rGO-EG fluids have shown stable uniform dispersion up to $120^{\circ} \mathrm{C}$ and have demonstrated consistent solar-thermal energy harvesting performance during repeated solar radiation at $\sim 110^{\circ} \mathrm{C}$.

Received 20th November 2018

Accepted 26th March 2019

DOI: $10.1039 / \mathrm{c} 8 \mathrm{ra09533g}$

rsc.li/rsc-advances eliminated the thermal resistance between the absorbing plate surface and the working fluids. ${ }^{15}$

Solar-thermal fluids are the core component in the DASC, and the thermophysical properties of the base fluids and solarabsorbing particles have significant influence on the solarthermal harvesting performance of the collector. ${ }^{15-18}$ Despite having been extensively studied, water-based fluids have a relatively narrow operation temperature range limited by the freezing and evaporation of water. By comparison, the EG-based fluids have a much broader operation temperature range from $-12.9^{\circ} \mathrm{C}$ to $197.3{ }^{\circ} \mathrm{C}$, which provides the possibility to use the fluids for medium-temperature industrial heating applications. ${ }^{19,20}$ Additionally, as shown by eqn (1), the energy conversion efficiency of $\operatorname{DASC}(\eta)$ is proportional to the temperature rise of the fluids. ${ }^{13,21}$

$$
\eta=\frac{m C_{\mathrm{p}}\left(T_{\mathrm{f}}-T_{\mathrm{i}}\right)}{Q_{\text {incident }}}
$$

where $m$ is the mass of the fluids, $C_{\mathrm{p}}$ is the heat capacity, and $T_{\mathrm{f}}$ and $T_{\mathrm{i}}$ are the final and initial temperature of the fluids, respectively. Such dependence implies that under the same solar illumination higher servicing temperature of the fluids is critical to achieve high conversion efficiency for the collector. Among various solar-absorbing particles investigated so far, ${ }^{11,22-24}$ carbon nanomaterials have been viewed as one of the most promising candidates due to their efficient broadband absorption of sunlight, which enables effective solar absorption through adding a minute amount of absorbers. The low loading requirement not only reduces the associated costs but
State Key Laboratory of Metal Matrix Composites, School of Materials Science and Engineering, Shanghai Jiao Tong University, 800 Dong Chuan Road, Shanghai 200240, P. R. China.E-mail: taopeng@sjtu.edu.cn; dengtao@sjtu.edu.cn

$\dagger$ Electronic supplementary information (ESI) available: Experimental section and supplementary figures. See DOI: 10.1039/c8ra09533g 
also helps maintain the high heat capacity and minimize the increase of viscosity of the fluids. A general issue that impedes the development and application of carbon nanofluids is their strong tendency to form aggregation. ${ }^{25,26}$ The aggregated fluids not only lose their volumetric solar absorption capability but also induces clogging and abrasion. In the past, introducing surface charge or surfactants ${ }^{27-33}$ or grafting with polymer chains $^{\mathbf{3 4 , 3 5}}$ had been adopted to mitigate the aggregation issue. While improved dispersion has been demonstrated within low-temperature water-based fluids, ${ }^{27-31,36-38}$ the dispersion stability of solar-thermal fluids under medium operation temperature is challenging and has been rarely investigated..$^{39-44}$ In particular, simple strategies rather than the complex and time-consuming surface treatment are highly desired to promote the practical applications of nanofluids for solar-thermal energy harvesting in the future. ${ }^{45}$

Herein, we reported a facile way to prepare EG-based fluids with stably dispersed reduced graphene oxide (rGO) for mediumtemperature solar-thermal energy harvesting. Specifically, as schemed by Fig. 1, we chose GO as the starting material and used ethanol as the intermediate dispersing solvent. By making use of the interaction between the oxygen-containing groups on the surface of GO and the ethanol molecules, we obtained ethanolwashed GO that can be homogeneously dispersed within EG fluids. Through a single-step heating process, GO is reduced into solar-absorbing rGO. The remaining oxygen-containing groups on the surface of rGO can further interact with the EG molecules, which results in the uniformly dispersed rGO-EG solar-thermal fluids. With the high specific heat and low viscosity similar to the base fluids, the obtained rGO-EG fluids showed significantly improved solar absorption and solar-thermal conversion capability over the EG base fluids. The prepared rGO-EG fluids have maintained their stable dispersion with a heating temperature up to $120{ }^{\circ} \mathrm{C}$ and have shown consistent solar-thermal harvesting performance during repeated solar heating and cooling cycles.

\section{Materials and methods}

\section{Materials}

Ethanol, sodium nitrate, potassium permanganate, ethylene glycol (EG) and concentrated sulfuric acid (98\%) were purchased from Sinopharm (Shanghai, China). Nano-graphite

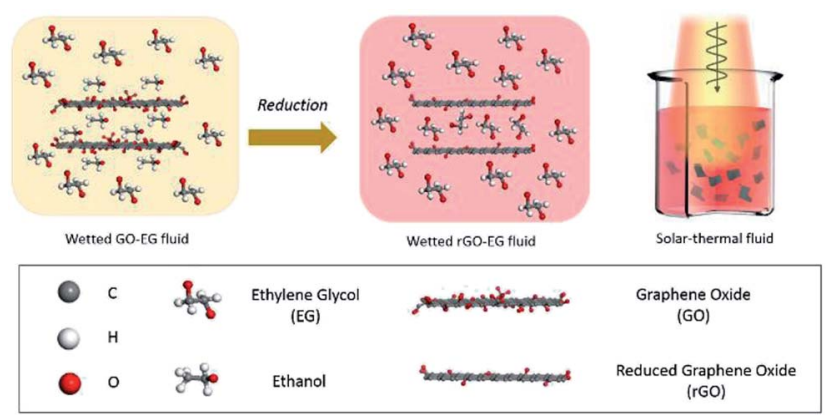

Fig. 1 Schematic of rGO-EG fluids for direct absorption solar-thermal energy harvesting. powders with a diameter $(\sim 400 \mathrm{~nm})$ were purchased from XFNano (Nanjing, China).

\section{Preparation of rGO-EG fluids}

GO was synthesized by the modified Hummers method. ${ }^{43}$ In a typical experiment, $40 \mathrm{~mL}$ of concentrated sulfuric acid (98\%) was added to the mixture of $0.5 \mathrm{~g}$ of nano-graphite powder and $0.5 \mathrm{~g}$ of sodium nitrate in a $500 \mathrm{~mL}$ round bottom flask. Then, $3 \mathrm{~g}$ of potassium permanganate was added to the solution that was placed within an ice bath. After that, the flask was transferred to a warm water bath at $40{ }^{\circ} \mathrm{C}$. After stirring for $1.5 \mathrm{~h}, 30 \mathrm{~mL}$ of deionized (DI) water was added to the mixture and the solution was stirred for another $30 \mathrm{~min}$. Subsequently, $100 \mathrm{~mL}$ of DI water was added following with dropwise addition of $3 \mathrm{~mL}$ of hydrogen peroxide. The dispersion was then subject to centrifugation at $1000 \mathrm{rpm}$ for $2 \mathrm{~min}$. After discarding the sediment, the collected solution was centrifuged at $8000 \mathrm{rpm}$ for $15 \mathrm{~min}$ and washed with water for 3 times. The as-prepared graphite oxide was soaked in $1: 10$ (by volume) $\mathrm{HCl}$ aqueous solution for $12 \mathrm{~h}$, then centrifuged at $8000 \mathrm{rpm}$ for $15 \mathrm{~min}$ and washed with ethanol for 5 times to remove residual $\mathrm{HCl}$. The centrifuged GO was dried in air at room temperature for $5 \mathrm{~h}$ before mixing with EG to form dispersion through sonication. The final rGO-EG fluids were obtained by heating the ethanol-washed GO-EG fluids at $120^{\circ} \mathrm{C}$ for $12 \mathrm{~h}$.

\section{Materials characterizations}

The water-washed and ethanol-washed graphite oxides are characterized by the Shimadzu X-ray diffractometer (LabX XRD6100). The morphology of fabricated monolayer GO sheet was checked with a transmission electron microscope (TEM, JEOL$2100 \mathrm{~F}$ ) operated at $200 \mathrm{kV}$. Atomic force microscopic (AFM) images of fabricated monolayer GO sheets were taken on an Atomic Force Microscopy (NT-MDT Prima Instruments, Russia). FTIR spectra were collected with a Fourier transform infrared spectrometer (Bruker Equinox 55). XPS spectra were recorded by an X-ray photoelectron spectroscopy (XPS, AXIS UltraDLD). Optical transmission spectra of each fluid sample were measured at room temperature by a UV-Vis-NIR spectrophotometer (PerkinElmer Lambda 330) using a quartz cuvette with an optical length of $10 \mathrm{~mm}$ as the container. Zeta potential $(\zeta)$ of fluids was measured by the Particle Sizer and Zeta Potential Analyzer (Omni Instruments, Japan). A differential scanning calorimeter (DSC, PerkinElmer DSC8000, TA Instruments) was used to measure the specific heat capacity. The temperature was programmed from $20^{\circ} \mathrm{C}$ to $80^{\circ} \mathrm{C}$ at a rising rate of $10^{\circ} \mathrm{C} \mathrm{min}{ }^{-1}$. The viscosity of each nanofluid sample was measured by a viscometer (Fangrui, LVDV-1T). The thermal conductivity of the EG and rGO-EG fluids was measured by a hot disk method (Hot disk 2500S). Each sample was tested for 10 times to obtain the average thermal conductivity.

\section{Results and discussion}

\section{Preparation of rGO-EG fluids}

Fig. 2 schematically shows the process for preparing homogeneously dispersed rGO-EG solar-thermal fluids. Nano-graphite 
was oxidized and exfoliated into GO by using the modified Hummers method. Considering the reported stability issue of GO within aqueous media, ${ }^{46}$ we adopted ethanol as the washing solvent to facilitate the subsequent mixing and dispersing within EG base fluid. The ethanol-washed graphite oxide was partially dried in air. With the assist of ultrasonication, the partly dried GO sheets can be readily redispersed within the EG fluid. The homogeneously dispersed rGO-EG fluid was obtained by simply heating the GO-EG fluid.

The XRD pattern in Fig. 3a shows that the partially dried ethanol-washed graphite oxide displays a diffraction peak at $9.4^{\circ}$, which corresponds to an interlayer distance of $0.94 \mathrm{~nm}$ according to the Bragg's diffraction law. In the control experiment, we also prepared graphite oxide by washing it with water and dried it in an oven. The resultant water-washed graphite oxide sample shows a diffraction peak at $11.3^{\circ}$, and the corresponding interlayer distance is $0.79 \mathrm{~nm}$. The increased spacing is due to the intercalation of ethanol molecules between GO sheets. ${ }^{46}$ Compared with water molecules, ethanol has a larger molecular volume but weaker interaction with the oxygencontaining groups on the GO surfaces. Thus, the ethanolwashed graphite oxide has a larger inter-plane distance than the water-washed one. The increased separation weakens the strong van der Waals attraction between adjacent GO layers, which helps exfoliate the graphite oxide into single-layered GO. The fabrication of monolayer GO was evidenced by the TEM observation in Fig. 3b and S1. $\dagger$ AFM measurement shown in Fig. $3 \mathrm{c}$ further supports the formation of single-layer ethanolwetted GO sheets. The thickness of the exfoliated GO sheets was measured to be $0.96 \pm 0.02 \mathrm{~nm}$, which indicates that the ethanol-wetted GO sheets are single-layered $(<1 \mathrm{~nm}$ in thickness). ${ }^{4-47}$ The ethanol-washed GO sheets can be readily dispersed within EG to form homogeneous dispersion.

Despite that the GO-EG fluids have formed uniform dispersion, they are yellow-colored (Fig. 4a), which means that they are not a good absorber for the broadband sunlight. To improve the solar absorption performance, we further converted them into black-colored rGO-EG fluids by heating the GO-EG fluids (Fig. 4a). The FTIR spectra in Fig. 4b show the presence of oxygenated groups on the surface of GO and rGO. Specifically, the stretching vibration peak of hydroxyl groups at $\sim 3420 \mathrm{~cm}^{-1}$, the stretching vibration peak of carbonyl groups at $\sim 1730 \mathrm{~cm}^{-1}$ and the vibration peak of epoxy groups at $\sim 1050 \mathrm{~cm}^{-1}$ were identified in both samples. By comparison, the intensity of vibration peaks of oxygen-containing groups was significantly decreased after reduction. Such reduced content of oxygencontaining groups was also confirmed by the XPS measurement. The C 1s XPS spectrum of GO in Fig. 4c shows four peaks at $284.6 \mathrm{eV}, 286.6 \mathrm{eV}, 288.7 \mathrm{eV}$, and $290.6 \mathrm{eV}$, corresponding to

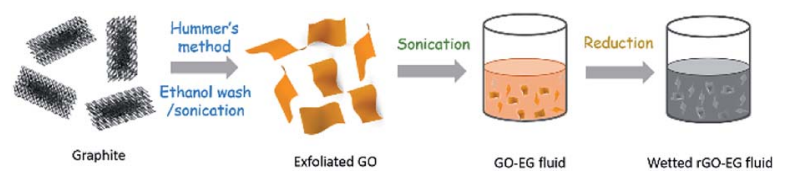

Fig. 2 Schematic processes for the preparation of dispersed rGO-EG solar-thermal fluids.

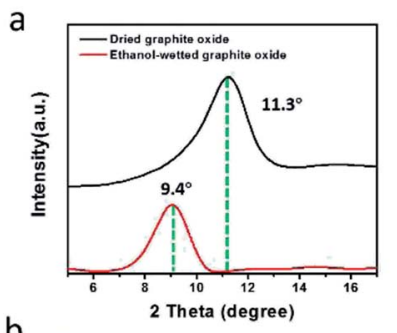

b
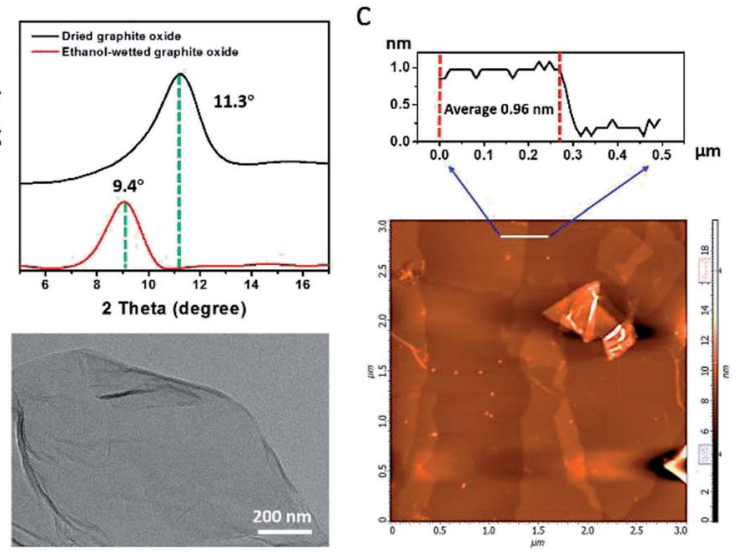

Fig. 3 (a) XRD pattern of water-washed and ethanol-washed graphite oxide; (b) TEM image of ethanol-washed GO; (c) AFM image and scanning profile of ethanol-washed GO.

$\mathrm{C}-\mathrm{C}, \mathrm{C}-\mathrm{O}, \mathrm{C}=\mathrm{O}$ and $\mathrm{O}-\mathrm{C}=\mathrm{O}$ binding states. ${ }^{48}$ Quantitative fitting of the spectrum indicates a relative content of $23.82 \%$, $52.5 \%, 18.96 \%$ and $4.72 \%$, respectively. Similarly, the C 1s XPS spectrum of rGO in Fig. 4d shows three peaks at $284.8 \mathrm{eV}, 286$. $4 \mathrm{eV}$ and $288.9 \mathrm{eV}$, which corresponds to $\mathrm{C}-\mathrm{C}, \mathrm{C}-\mathrm{O}$ and $\mathrm{C}=\mathrm{O}$ binding, respectively. These three binding states account $50.9 \%, 38.95 \%$ and $10.15 \%$, respectively. The reduced peak intensity of $\mathrm{C}-\mathrm{O}$ and $\mathrm{C}=\mathrm{O}$ binding states indicates that the $\mathrm{GO}$ had been partially reduced.

We comparatively prepared the EG-based solar-thermal fluids $\left(1.6 \mathrm{mg} \mathrm{mL}^{-1}\right)$ by dispersing water-washed $\mathrm{rGO}$ and ethanol-washed GO into EG. As shown in Fig. S2, $\dagger$ although they initially have the similar homogeneous dispersion, only the EG fluids dispersed with ethanol-washed GO have maintained the stable dispersion after heating at $120^{\circ} \mathrm{C}$ for $12 \mathrm{~h}$, which highlights the importance of separated inter-plane distance for achieving uniform dispersion. In another controlled experiment, we mixed the same ethanol-washed GO in Dowtherm A oil, another commonly used high-temperature base fluid, to prepare the solar-thermal fluids. It turned out that after heating
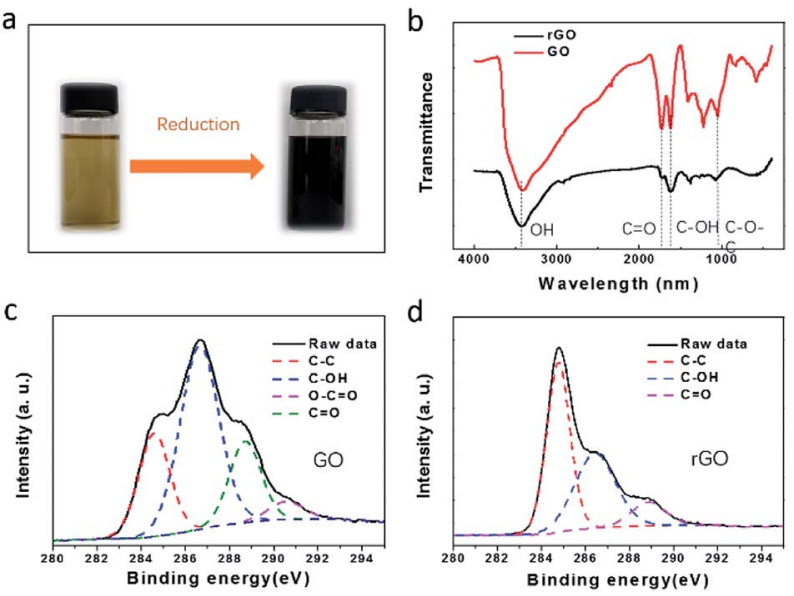

Fig. 4 (a) Photographs of ethanol-washed GO-EG fluids before and after reduction; (b) FTIR spectra of GO and rGO; (c) C 1s XPS spectra of GO; (d) C 1s XPS spectra of rGO. 
the rGO sheets formed aggregates and precipitated out of the base fluid. These results show that the polar EG molecules can interact with the surface oxygen-containing groups on the surface of rGO to achieve uniform dispersion, but it lacks such favorable interaction between rGO and the Dowtherm A oil.

\section{Thermophysical properties of rGO-EG fluids}

To fulfill the requirement for high-performance solar-thermal energy harvesting applications, the fluids should have good absorption of sunlight, high specific heat capacity, and low viscosity. We further characterized the physical properties of the rGO-EG fluids with different loadings $\left(0.2 \mathrm{mg} \mathrm{mL}^{-1}, 0.4 \mathrm{mg}\right.$ $\mathrm{mL}^{-1}, 0.8 \mathrm{mg} \mathrm{mL}{ }^{-1}, 1.6 \mathrm{mg} \mathrm{mL}^{-1}$ ). We measured the optical transmittance of the rGO-EG fluids over the solar irradiation spectral wavelengths by using air as the reference. Fig. 5 a shows that the transmittance of rGO-EG fluids rapidly decreases with increasing loading of rGO. With a loading of $1.6 \mathrm{mg} \mathrm{mL}^{-1}$, the rGO-EG fluids almost fully absorb the incident light ranging from 250 to $2500 \mathrm{~nm}$. To better characterize the absorption performance of the fluids, as shown in Fig. S3, $\dagger$ we calculated the extinction coefficient $(\alpha)$ by converting the transmittance $(T)$ according to the Beer-Lambert law:

$$
\alpha=-\frac{1}{l} \ln (T)
$$

where $l$ is the optical length $(1 \mathrm{~cm})$. Based on the measured extinction coefficient, we further calculated the penetration depth of sunlight $(x)$ within the rGO-EG fluids by eqn (3): ${ }^{49}$

$$
F(x)=1-\frac{\int_{\lambda_{\min }}^{\lambda_{\max }} I_{\lambda} \mathrm{e}^{-\alpha x} \mathrm{~d}(\lambda)}{\int_{\lambda_{\min }}^{\lambda_{\max }} I_{\lambda} \mathrm{d}(\lambda)}
$$

where $F(x)$ is the fraction of stored solar-thermal energy within solar-thermal fluids after passing a distance of $x$ across the fluids, $I_{\lambda}$ is the incident solar irradiance, $\lambda_{\max }$ and $\lambda_{\min }$ are the maximum and minimum wavelength of the solar spectrum.
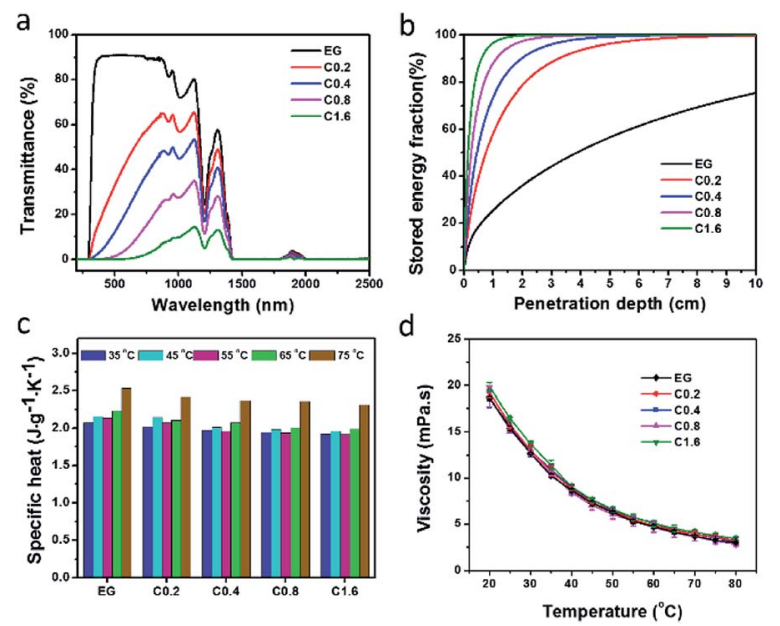

Fig. 5 (a) Transmittance spectra of EG and $\mathrm{rGO}$-EG fluids with different loading; (b) penetration depth of EG and rGO-EG fluids; (c) specific heat capacity of EG and rGO-EG fluids at different temperatures; (d) viscosity of EG and $\mathrm{rGO}-\mathrm{EG}$ fluids as a function of temperature.
Fig. 5b shows that the incident sunlight is quickly absorbed by a thin layer of rGO-EG fluids and with increasing loading of rGO the penetration depth becomes shorter. For the $1.6 \mathrm{mg} \mathrm{mL}^{-1}$ rGO-EG fluids, the incident solar light is fully absorbed within a penetration depth of $2 \mathrm{~cm}$, whereas the pure EG fluids only absorb less than $40 \%$ of incident solar energy for the same penetration depth, indicating significantly enhanced solarthermal harvesting capability by the added rGO sheets.

Fig. $5 \mathrm{c}$ presents that the specific heat capacity of these rGOEG fluids increases with increasing temperature due to stronger molecular vibration contribution. At the same time, it shows that adding rGO only leads to negligible decrease of specific heat capacity of the fluids due to the low loading of homogeneous dispersed rGO (Table S1 $\dagger$ ). Viscosity is another important parameter for solar-thermal fluids, which directly influences the pumping pressure when the fluids are in circulation. Fig. 5d and Table $\mathrm{S} 2 \uparrow$ show that the viscosity of the rGO-EG fluids decreases with increasing temperature and it varies little with the increasing loading of rGO. Compared to the pure EG fluid, the unchanged viscosity of rGO-EG fluid is due to both the low loading of rGO and its homogenous dispersion within EG. As described by eqn (4), the viscosity of the mixture system can be calculated by the classical Einstein theory: ${ }^{50}$

$$
\eta=\eta_{0}(1+2.5 \varphi)
$$

where $\eta$ is the viscosity of the nanofluid, $\eta_{0}$ is the viscosity of the base fluid, and $\varphi$ is volume fraction of nanoparticle. The densities of GO and EG are estimated to be $1.8 \mathrm{~g} \mathrm{~cm}^{-3}$, and $1.11 \mathrm{~g} \mathrm{~cm}^{-3}$, respectively. For the rGO-EG fluids with a loading of $1.6 \mathrm{mg} \mathrm{mL}^{-1}$, the calculated viscosity of the nanofluids is only $0.22 \%$ higher than the base fluid. We also measured the thermal conductivity of EG and rGO-EG fluids at room temperature. Table $\mathrm{S} 3 \uparrow$ shows slight increase of thermal conductivity from $0.2599 \pm 0.0058 \mathrm{~W} \mathrm{~m}^{-1} \mathrm{~K}^{-1}$ for the EG base fluids to $0.2674 \pm 0.007 \mathrm{~W} \mathrm{~m}^{-1} \mathrm{~K}^{-1}$ for the rGO-EG fluids $\left(1.6 \mathrm{mg} \mathrm{mL} \mathrm{m}^{-1}\right)$.

\section{Dispersion stability of rGO-EG fluids}

Maintaining uniform dispersion of the rGO-EG fluids is the key to purse their consistent solar-thermal applications. We first assessed their static dispersion stability against sedimentation at room temperature. As shown by the photographs in Fig. 6a and $\mathrm{b}$, the rGO-EG fluids have demonstrated the same homogeneous dispersion without observing any visible precipitation after being stored for two weeks. Fig. $6 \mathrm{c}$ presents that the transmittance spectra of the fluids after storing for one and two weeks are almost overlapped with that of the as-prepared fluids, which also confirmed the stable dispersion of the rGO-EG fluids.

After confirming the stable dispersion of rGO-EG fluids at room temperature, we further evaluated their dispersion behavior at elevated temperatures. Considering that typically the available solar illumination time for daily solar-thermal energy harvesting is no more than $12 \mathrm{~h}$, here we choose $12 \mathrm{~h}$ as the representative heating duration to evaluate the dispersion stability of the solar-thermal fluids. The rGO-EG fluids with 

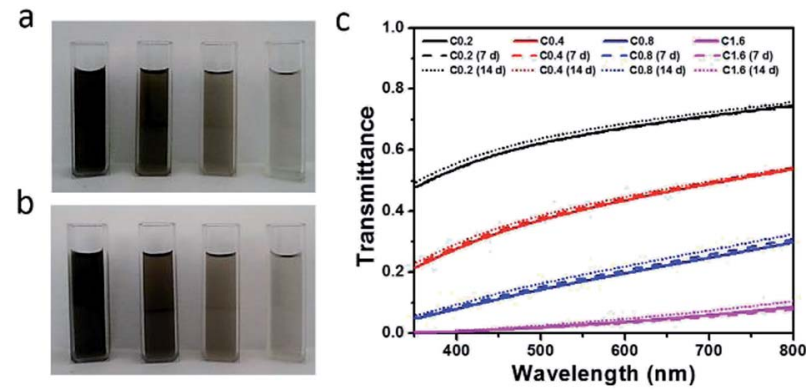

Fig. 6 (a) Photographs of as-prepared rGO-EG fluids with different loadings (from left to right: $1.6 \mathrm{mg} \mathrm{mL}^{-1}, 0.8 \mathrm{mg} \mathrm{mL}^{-1}, 0.4 \mathrm{mg} \mathrm{mL}^{-1}$, $0.2 \mathrm{mg} \mathrm{mL}^{-1}$ ); (b) photographs of rGO-EG fluids after being stored at room temperature for two weeks; (c) transmittance spectra of $\mathrm{rGO}-\mathrm{EG}$ fluids before and after being stored at room temperature for one week (7 d) and two weeks (14 d).

different loadings were heated at $60^{\circ} \mathrm{C}, 90^{\circ} \mathrm{C}, 120^{\circ} \mathrm{C}$ and $150^{\circ} \mathrm{C}$ for $12 \mathrm{~h}$. As shown by the photographs in Fig. $\mathrm{S} 4, \uparrow$ there were no visible aggregation in fluids after heating at $60{ }^{\circ} \mathrm{C}, 90{ }^{\circ} \mathrm{C}$ and $120{ }^{\circ} \mathrm{C}$, but when the temperature was raised to $150{ }^{\circ} \mathrm{C}$ the rGO particles were aggregated and partly precipitated on the wall of the quartz cuvette. The transmittance spectra of fluids in Fig. 7 show the same trend. For the sample heated at $60{ }^{\circ} \mathrm{C}$, the transmittance spectra of fluids before and after heating are almost overlapped (Fig. 7a). For the fluids that are heated at $90{ }^{\circ} \mathrm{C}$ and $120^{\circ} \mathrm{C}$, Fig. $7 \mathrm{~b}$ and $\mathrm{c}$ show that there was slight drop in the transmittance after heating, which is due to further reduction of rGO during the heating process. In contrast, Fig. $7 \mathrm{~d}$ shows that the transmittance of the fluids sharply increases after heating at $150{ }^{\circ} \mathrm{C}$ as a result of precipitation of the solarabsorbing rGO sheets to the bottom of the cuvette. The photographs in Fig. $\mathrm{S} 4 \uparrow$ confirm the precipitation of rGO after heating at $150{ }^{\circ} \mathrm{C}$, so the incident light can easily penetrate through the fluids leading to high transmittance.

It can be seen that heating temperature has a profound influence on the stability of rGO-EG fluids and the fluids can keep stable dispersion at temperatures up to $120{ }^{\circ} \mathrm{C}$. The favorable interaction between the oxygen-containing functional groups on the surface of rGO sheets and the polar EG fluids is the main driving force for forming stable and homogeneous dispersion. With increased heating temperatures, the rGO sheets in the fluids would be further reduced and the amount of remained oxygen-containing groups will be decreased to such an extent that the attractive interaction between the rGO surface and the EG molecules is negligible. Without the adsorbed EG molecules on the surface, the strong inter-plane attraction between neighboring rGO sheets dominates and leads to aggregation. Zeta potential is an important parameter to analyze the dispersion stability of thermal fluids. Generally, the fluids with absolute zeta potential values greater than $25 \mathrm{mV}$ typically have high stability, and for those fluids with an absolute zeta potential above $30 \mathrm{mV}$ the dispersion is highly stable. ${ }^{45,51,52}$ As shown in Table $\mathrm{S} 1, \dagger$ the absolute zeta potential of the rGO-EG fluids before and after heating at $120{ }^{\circ} \mathrm{C}$ for $12 \mathrm{~h}$ was measured to be $31.42 \mathrm{mV}$ and $25.70 \mathrm{mV}$, respectively. By comparison, after heating at $150{ }^{\circ} \mathrm{C}$ for $12 \mathrm{~h}$ the absolute zeta
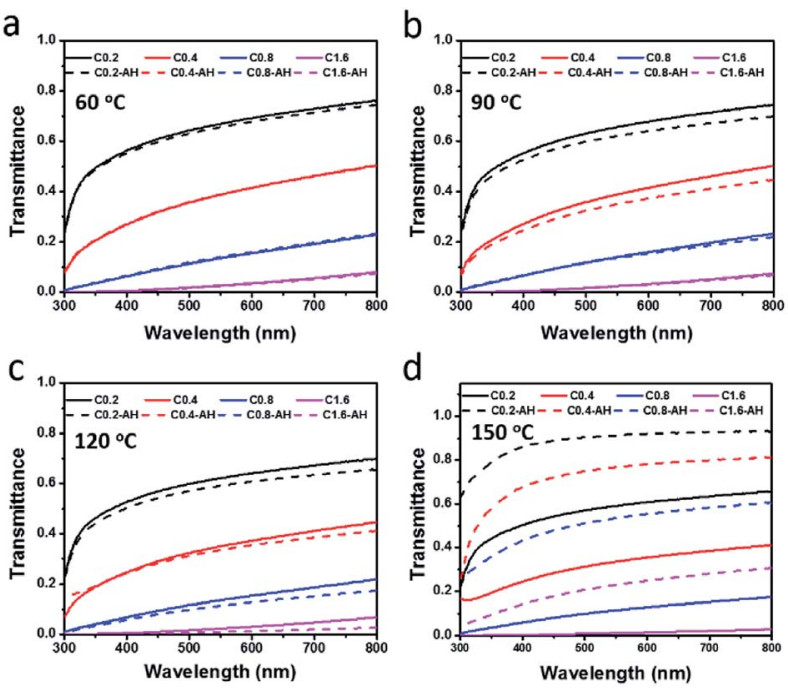

Fig. 7 Transmittance spectra of rGO-EG fluids with different loadings before (solid lines) and after (dashed lines) $12 \mathrm{~h}$ continuous heating at different temperatures: (a) $60^{\circ} \mathrm{C}$, (b) $90^{\circ} \mathrm{C}$, (c) $120{ }^{\circ} \mathrm{C}$, (d) $150{ }^{\circ} \mathrm{C}$.

potential of the rGO-EG fluids decreased to $14.76 \mathrm{mV}$ due to the removal of polar oxygen-containing groups at the rGO surface.

\section{Solar-thermal harvesting by rGO-EG fluids}

With the obtained stable dispersion of rGO-EG fluids, we evaluated their solar-thermal harvesting performances. As shown in Fig. S5, $\uparrow$ we measured the temperature evolution profiles of the fluids by placing 4 thermocouples along the cuvette and all thermocouples were shielded by embedding them within the sidewall of the container. Fig. 8a shows that directly shedding light on the fluids causes a gradient temperature distribution along the height of the cuvette. After one-sun solar illumination for $1 \mathrm{~h}$ while the top portion of the fluid was heated to $52.5^{\circ} \mathrm{C}$ the bottom section was only heated to $40.0^{\circ} \mathrm{C}$. This temperature difference $\left(12.5{ }^{\circ} \mathrm{C}\right)$ is attributed to the limited penetration depth of the solar light in the rGO-EG fluids. Based on the calculation shown in Fig. 5b, the incident light was fully absorbed by the rGO-EG fluids within $2 \mathrm{~cm}$. Thus, the fluids beneath relies on heat diffusion from the top to be heated up. Such temperature difference can be eliminated by magnetically stirring the fluids. As evidenced by the overlapped temperature curves in Fig. 8b, the stirred fluids were uniformly heated.

We also investigated the impact of rGO loading on the solarthermal harvesting behavior of the stirred rGO-EG fluids. Fig. $8 \mathrm{c}$ shows that with the same one-sun illumination the solarheating temperatures increase with increasing loading of rGO. After illumination for $1 \mathrm{~h}$ pure EG only was heated to $29.2^{\circ} \mathrm{C}$, but the $1.6 \mathrm{mg} \mathrm{mL}^{-1}$ rGO-EG fluid has reached $49.6{ }^{\circ} \mathrm{C}$. The enhanced solar-thermal conversion with increasing loading of rGO is more clearly shown by the hourly temperature rise in Fig. 8d. Based on the measured temperature profiles in Fig. 8c, we further calculated the photothermal efficiency of EG and rGO-EG fluids under $1 \mathrm{~kW} \mathrm{~m}{ }^{-2}$ solar irradiation by using eqn (1). Fig. S6† shows that minute addition of rGO into the EG base fluids can significantly improve the photothermal conversion 

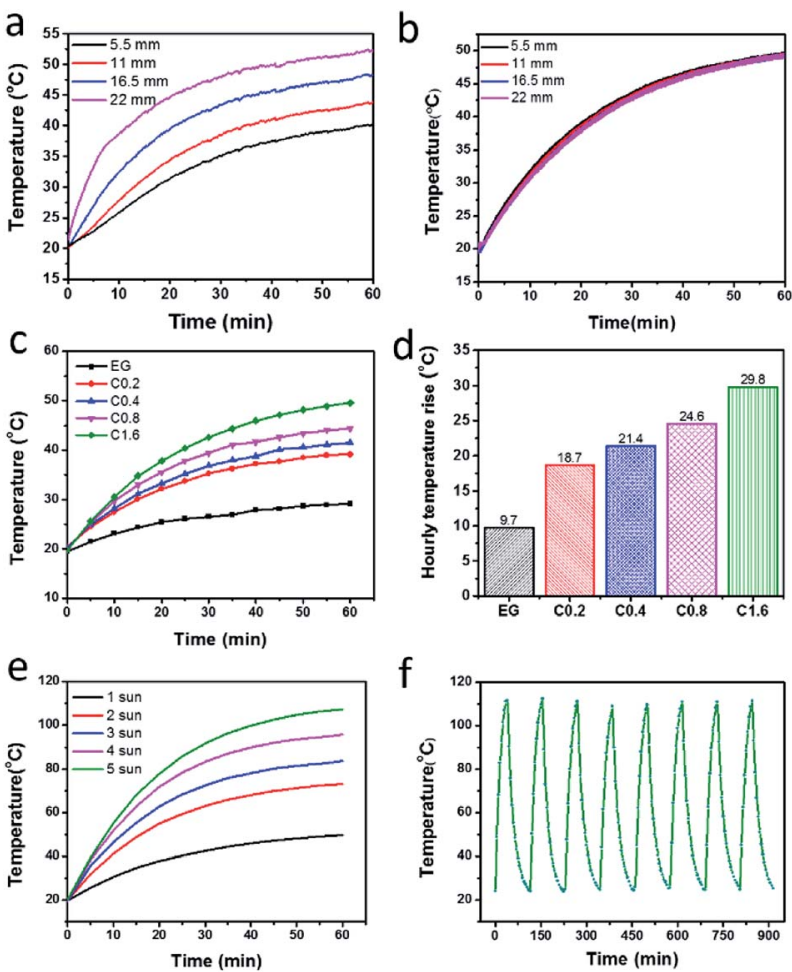

Fig. 8 (a) Temperature profiles of rGO-EG fluids (1.6 mg mL $\mathrm{mL}^{-1}$ ) measured at different heights under one-sun illumination without stirring; (b) temperature evolution profiles of rGO-EG fluids $\left(1.6 \mathrm{mg} \mathrm{mL}^{-1}\right)$ with magnetic stirring; (c) temperature profiles of $\mathrm{rGO}$-EG fluids with different loadings under one-sun illumination with magnetic stirring; (d) hourly temperature rise of the stirred rGO-EG fluids under one-sun illumination; (e) temperature profiles of the stirred rGO-EG fluids $(1.6 \mathrm{mg}$ $\mathrm{mL}^{-1}$ ) under different solar illumination intensity; (f) cycling performance of the $\mathrm{rGO}$-EG fluids $\left(1.6 \mathrm{mg} \mathrm{mL}^{-1}\right)$ under $6 \mathrm{~kW} \mathrm{~m}^{-2}$ solar irradiation.

efficiency. We also studied the effect of solar intensity on solarthermal conversion behavior of the fluids (Fig. 8e). After solar irradiation for $1 \mathrm{~h}$, the final temperature of the rGO-EG fluids continuously increases with stronger solar illumination and the $1.6 \mathrm{mg} \mathrm{mL}{ }^{-1}$ fluids was heated to $107.3{ }^{\circ} \mathrm{C}$ with 5 -sun illumination. Under the strong solar illumination, the photothermal efficiency of the rGO-EG fluids gradually dropped to $30 \%$ (Fig. S6 $\dagger$ ). As the photothermal efficiency of the receiver is dependent on solar concentration, nanofluid height, optical thickness and thermal insulation of the system,${ }^{17}$ it is expected that receiver efficiency at medium operation temperatures can be further improved by optimizing these factors. The cycling stability of the fluids was tested by repeated heating the $1.6 \mathrm{mg}$ $\mathrm{mL}^{-1}$ rGO-EG fluids to $110{ }^{\circ} \mathrm{C}$ through strong solar irradiation $\left(6 \mathrm{~kW} \mathrm{~m}^{-2}\right)$ for $40 \mathrm{~min}$ followed by naturally cooling down the fluids to room temperature by $75 \mathrm{~min}$. Fig. 8 f shows that the prepared rGO-EG fluids have maintained their stable solarthermal heating performance during continuous heating and cooling for 8 cycles.

\section{Conclusions}

In summary, we reported a simple method to fabricate homogenously dispersed rGO-EG fluids for direct solar-thermal energy harvesting at medium temperatures. Instead of resorting to complex surface modification treatment of graphene, we selected GO as the starting material and made use of the interaction between the oxygen-containing groups at GO surfaces and the polar ethanol dispersing solvent to prepare ethanol-washed GO that is miscible with EG fluids. After a single step of heating, $\mathrm{GO}$ was transformed into $\mathrm{rGO}$ and the EG molecules directly interact with the remaining oxygencontaining groups at the rGO surface as the ethanol evaporates. Compared with the EG base fluids, the obtained rGO-EG fluids have significantly improved broadband absorption of sunlight whiling maintaining similar high specific heat and low viscosity, which would make them an attractive solar-thermal fluid for the DASC systems operating at medium temperatures. It is also expected that similar facile fabrication strategy could be applied to disperse rGO within other functional fluids for diverse applications. Ideally, the potential medium-to-high temperature solar-thermal fluids also need to have low emissivity in order to suppress the radiation heat losses, but the EG base fluids and the rGO-EG fluids have shown the same high emissivity of 0.97 . To this end, both low-emissivity base fluids and nanoscale low-emissivity solar-thermal converters similar to the commercial selective solar-thermal coatings should be developed.

\section{Conflicts of interest}

There are no conflicts to declare.

\section{Acknowledgements}

The authors thank the financial support from National Key R\&D Program of China (2016YFB0402100), National Natural Science Foundation of China (Grant No: 51873105, 51403127, 51521004 and 51420105009), Shanghai Rising-Star Program (Grant No: 18QA1402200), Innovation Program of Shanghai Municipal Education Commission (Grant No. 2019-01-07-00-02-E00069) and "Chen Guang" project from Shanghai Municipal Education Commission and Shanghai Education Development Foundation under Grant No. 15CG06. We also thank the support from Science and Technology on Monolithic Integrated Circuits and Modules Laboratory (614280303020317) and Interdisciplinary Program of Shanghai Jiao Tong University (YG2015QN19, YG2017QN68).

\section{References}

1 S. Chu, Y. Cui and N. Liu, Nat. Mater., 2017, 16, 16-22.

2 G. W. Crabtree and N. S. Lewis, Phys. Today, 2007, 60, 37-42.

3 A. Argiriou, N. Klitsikas, C. A. Balaras and D. N. Asimakopoulos, Energy and Buildings, 1997, 26, 215221.

$4 \mathrm{M}$. Thirugnanasambandam, S. Iniyan and R. Goic, Renewable Sustainable Energy Rev., 2010, 14, 312-322.

5 P. Tao, G. Ni, C. Song, W. Shang, J. Wu, J. Zhu, G. Chen and T. Deng, Nat. Energy, 2018, 3, 1031-1041. 
6 H. Ghasemi, G. Ni, A. M. Marconnet, J. Loomis, S. Yerci, N. Miljkovic and G. Chen, Nat. Commun., 2014, 5, 5449.

7 S. A. Kalogirou, Prog. Energy Combust. Sci., 2004, 30, 231-295. 8 Z. Y. Wang, Z. Tong, Q. X. Ye, H. Hu, X. Nie, C. Yan, W. Shang, C. Y. Song, J. B. Wu, J. Wang, H. Bao, P. Tao and T. Deng, Nat. Commun., 2017, 8, 1478.

9 C. Chang, C. Yang, Y. M. Liu, P. Tao, C. Y. Song, W. Shang, J. B. Wu and T. Deng, ACS Appl. Mater. Interfaces, 2016, 8, 23412-23418.

10 B. Y. Liu and R. C. Jordan, Sol. Energy, 1963, 7, 53-74.

11 G. Ni, N. Miljkovic, H. Ghasemi, X. P. Huang, S. V. Boriskina, C. T. Lin, J. J. Wang, Y. F. Xu, M. M. Rahman, T. J. Zhang and G. Chen, Nano Energy, 2015, 17, 290-301.

12 J. E. Minardi and H. N. Chuang, Sol. Energy, 1975, 17, 179183.

13 H. Tyagi, P. Phelan and R. Prasher, J. Sol. Energy Eng., 2009, 131, 143-155.

14 T. P. Otanicar, P. E. Phelan, R. S. Prasher, G. Rosengarten and R. A. Taylor, J. Renewable Sustainable Energy, 2010, 2, 033102.

15 T. B. Gorji and A. A. Ranjbar, Renewable Sustainable Energy Rev., 2017, 72, 10-32.

16 A. Kasaeian, A. T. Eshghi and M. Sameti, Renewable Sustainable Energy Rev., 2015, 43, 584-598.

17 A. Lenert and E. N. Wang, Sol. Energy, 2012, 86, 253-265.

18 W. J. Chen, C. J. Zou and X. K. Li, Sol. Energy Mater. Sol. Cells, 2017, 163, 157-163.

19 S. Kalogirou, Appl. Energy, 2003, 76, 337-361.

20 D. N. Nkwetta, M. Smyth, A. Zacharopoulos and T. Hyde, Int. J. Energy Res., 2013, 37, 570-581.

21 R. A. Taylor, P. E. Phelan, T. P. Otanicar, C. A. Walker, M. Nguyen, S. Trimble and R. Prasher, J. Renewable Sustainable Energy, 2011, 3, 4.

22 O. Neumann, C. Feronti, A. D. Neumann, A. J. Dong, K. Schell, B. Lu, E. Kim, M. Quinn, S. Thompson, N. Grady, P. Nordlander, M. Oden and N. J. Halas, Proc. Natl. Acad. Sci. U. S. A., 2013, 110, 11677-11681.

23 O. Neumann, A. S. Urban, J. Day, S. Lal, P. Nordlander and N. J. Halas, ACS Nano, 2013, 7, 42-49.

24 E. Sani, L. Mercatelli, S. Barison, C. Pagura, F. Agresti, L. Colla and P. Sansoni, Sol. Energy Mater. Sol. Cells, 2011, 95, 2994-3000.

25 F. Yu, Y. Y. Chen, X. B. Liang, J. L. Xu, C. S. Lee, Q. Liang, P. Tao and T. Deng, Prog. Nat. Sci., 2017, 27, 531-542.

26 S. Mesgari, R. A. Taylor, N. E. Hjerrild, F. Crisostomo, Q. Y. Li and J. Scott, Sol. Energy Mater. Sol. Cells, 2016, 157, 652-659.

27 R. Shende and R. Sundara, Sol. Energy Mater. Sol. Cells, 2015, 140, 9-16.

28 S. H. Lee and S. P. Jang, Int. J. Heat Mass Transfer, 2015, 80, 58-71.

29 L. L. Chen, C. Xu, J. Liu, X. M. Fang and Z. G. Zhang, Sol. Energy, 2017, 148, 17-24.

30 L. L. Chen, J. Liu, X. M. Fang and Z. G. Zhang, Sol. Energy Mater. Sol. Cells, 2017, 163, 125-133.
31 S. Khosrojerdi, A. M. Lavasani and M. Vakili, Sol. Energy Mater. Sol. Cells, 2017, 164, 32-39.

32 L. H. Kong, J. L. Sun and Y. Y. Bao, RSC Adv., 2017, 7, 1259912609.

33 H. K. Arzani, A. Amiri, S. N. Kazi, A. Badarudin and B. T. Chew, RSC Adv., 2016, 6, 65654-65669.

34 H. K. He and C. Gao, Chem. Mater., 2010, 22, 5054-5064.

35 Y. Y. Chen, X. J. Quan, Z. Y. Wang, C. S. Lee, Z. Z. Wang, P. Tao, C. Y. Song, J. B. Wu, W. Shang and T. Deng, J. Mater. Chem. A, 2016, 4, 17503-17511.

36 M. Vakili, S. M. Hosseinalipour, S. Delfani, S. Khosrojerdi and M. Karami, Sol. Energy, 2016, 131, 119-130.

37 S. Iranmanesh, H. C. Ong, B. C. Ang, E. Sadeghinezhad, A. Esmaeilzadeh and M. Mehrali, J. Cleaner Prod., 2017, 162, 121-129.

38 S. K. Verma, A. K. Tiwari and D. S. Chauhan, Energy Convers. Manage., 2017, 134, 103-115.

39 N. Hordy, D. Rabilloud, J. L. Meunier and S. Coulombe, Sol. Energy, 2014, 105, 82-90.

40 M. Bortolato, S. Dugaria, F. Agresti, S. Barison, L. Fedele, E. Sani and D. Del Col, Energy Convers. Manage., 2017, 150, 693-703.

41 J. Liu, Z. C. Ye, L. Zhang, X. M. Fang and Z. G. Zhang, Sol. Energy Mater. Sol. Cells, 2015, 136, 177-186.

42 R. Gomez-Villarejo, J. Navas, E. I. Martin, A. SanchezCoronilla, T. Aguilar, J. J. Gallardo, D. De los Santos, R. Alcantara, C. Fernandez-Lorenzo and J. Martin-Calleja, J. Mater. Chem. A, 2017, 5, 12483-12497.

43 P. Tao, L. Shu, J. Zhang, C. Lee, Q. Ye, H. Guo and T. Deng, Prog. Nat. Sci., 2018, 28, 554-562.

44 J. Liu, F. X. Wang, L. Zhang, X. M. Fang and Z. G. Zhang, Renewable Energy, 2014, 63, 519-523.

45 L. Dong, Z. X. Chen, X. X. Zhao, J. H. Ma, S. Lin, M. X. Li, Y. Bao, L. Q. Chu, K. Leng, H. B. Lu and K. P. Loh, Nat. Commun., 2018, 9, 76.

46 W. C. Du, M. M. Wu, M. Zhang, G. C. Xu, T. T. Gao, L. Qian, X. W. Yu, F. Y. Chi, C. Li and G. Q. Shi, Chem. Commun., 2017, 53, 11005-11007.

47 X. L. Li, X. R. Wang, L. Zhang, S. W. Lee and H. J. Dai, Science, 2008, 319, 1229-1232.

48 S. F. Pei, J. P. Zhao, J. H. Du, W. C. Ren and H. M. Cheng, Carbon, 2010, 48, 4466-4474.

49 E. Sani, S. Barison, C. Pagura, L. Mercatelli, P. Sansoni, D. Fontani, D. Jafrancesco and F. Francini, Opt. Express, 2010, 18, 5179-5187.

50 M. K. Abdolbaqi, N. A. C. Sidik, A. Aziz, R. Mamat, W. H. Azmi, M. N. A. W. M. Yazid and G. Najafi, Int. Commun. Heat Mass Transfer, 2016, 77, 22-32.

51 W. Yu and H. Q. Xie, J. Nanomater., 2012, 1, 1687-4110.

52 M. Vakili, S. M. Hosseinalipour, S. Delfani and S. Khosrojerdi, Sol. Energy Mater. Sol. Cells, 2016, 152, 187191. 